\title{
LA STRUTTURAZIONE DEI SISTEMI PARTITICI NELLE NUOVE DEMOCRAZIE
}

\author{
di Maurizio Cotta
}

Sistemi partitici e spazio politico nelle nuove democrazie

La ricostruzione di un sistema partitico competitivo dopo la caduta di un regime non democratico (che per definizione aveva soppresso il pluralismo partitico) non comporta soltanto la creazione (e poi eventualmente il consolidamento) di un numero maggiore o minore di partiti con le proprie identità ideologiche, programmatiche e organizzative, ma anche la definizione di uno spazio politico all'interno del quale queste unità partitiche interagiranno. In effetti nei sistemi politici consolidati le identità stesse delle singole unità partitiche possono essere pienamente determinate solo collocandole nella dimensione sistemica.

La nozione di spazio politico (Downs 1957; Sartori 1976; Budge e Keman 1990) è rilevante tanto per il livello di massa che per il livello di élite della politica. Il primo livello riguarda la collocazione dei partiti e degli elettori nella competizione elettorale. Qui gli interrogativi principali riguardano il carattere unimodale o polimodale della distribuzione degli elettori, il numero delle dimensioni richieste per interpretare il posiziona-

Una prima versione di questo articolo con il titolo The new party systems after the dictatorship. Coalitions, alliances, fusions and party splits during the transition and post-transition stages $\dot{e}$ stata presentata al convegno «The emergence of new party systems and transitions to democracy: inter-regional comparisons between eastern and southern Europe» organizzato dal Centre for Mediterranean Studies della Università di Bristol (17-19 Settembre 1993). La versione inglese con il titolo Coalitions. Alliances, Fusions and Party Splits during the Transition and Post-Transition Stages verrà pubblicata nel volume curato da P. Lewis $e$ G. Pridham, The Emergence of new Party Systems and Transitions to Democracy: Interregional Comparisons between Eastern and Southern Europe, London, Routledge, 1995. Per la ricostruzione degli avvenimenti nell'Europa Centro-orientale ho fatto largo uso dei «Research Reports of Radio Free Europe and Radio Liberty». La ricerca si è avvalsa dei Fondi $60 \%$ dell'Università di Siena.

RIVISTA ITALIANA DI SCIENZA POLITICA / a. XXV, n. 2, agosto 1995 
mento reciproco dei partiti, il grado maggiore o minore di rigidità degli elettori e dei partiti nel muoversi all'interno dello spazio di competizione. Il secondo livello riguarda piuttosto le relazioni tra i partiti nelle sedi istituzionali e, quindi, i vincoli e le opportunità posti ai partiti nei processi di costruzione delle coalizioni di governo e parlamentari. Qui il punto essenziale è quali partiti abbiano tra loro un potenziale di coalizione e quali no. Se vogliamo capire i nuovi sistemi politici emersi dal fallimento dei regimi non democratici, le caratteristiche dello spazio sistemico sono dunque altrettanto importanti che quelle delle singole unità partitiche.

In questo saggio cercherò di discutere appunto le interazioni fra costruzione dei partiti e strutturazione dello spazio politico durante gli anni formativi delle nuove democrazie. Lo farò utilizzando casi empirici tratti dalle tre ondate di democratizzazione che hanno avuto luogo in Europa rispettivamente dopo la seconda guerra mondiale, negli anni settanta e dopo la fine degli anni ottanta ${ }^{1}$. Il punto di partenza per questa discussione è dato dalla differenza tra una democrazia consolidata ed una di nuova data. Nella prima le caratteristiche dello spazio politico sono essenzialmente un dato. A meno naturalmente dell'evento piuttosto raro di una grave crisi, esse cambiano solo marginalmente e gradualmente. Ben diverso il caso di una nuova democrazia. Sotto il regime non democratico ovviamente non esisteva uno spazio politico vero e proprio data la mancanza di competizione. Quando la transizione alla democrazia reintroduce il pluralismo partitico lo spazio sistemico ed i suoi caratteri devono essere definiti da zero (o, eventualmente, sulla base del recupero di elementi di una precedente esperienza democratica). Le conseguenze sono rilevanti. In una democrazia consolidata le unità partitiche sono fortemente vincolate nella loro azione dalle caratteristiche strutturali dello spazio politico. D'altro canto queste contribuiscono a stabilizzare la forza dei partiti. Viceversa, in una democrazia nuova i partiti sono molto più esposti alle incertezze dell'ambiente, dato che appunto manca una struttura ben definita dello spazio sistemico; nello stesso tempo con la loro azione partecipano alla sua definizione in misura ben mag-

${ }^{1}$ La prima ondata riguarda Austria, Francia, Germania e Italia, alle quali si dovrebbero aggiungere, ma per un periodo molto breve, i paesi dell'Europa centro-orientale; la seconda i tre paesi dell'Europa meridionale (Grecia, Portogallo e Spagna); la terza tutti i paesi del blocco comunista. 
giore di quanto non avvenga in una democrazia consolidata. $\mathrm{Ne}$ consegue che la politica è un gioco molto più rischioso nelle democrazie giovani: la possibilità per le forze politiche di trovarsi «fuori mercato» è infatti assai concreta. Anche per questo le qualità della leadership (in specie la capacità di imboccare strade inesplorate) vi hanno un'importanza del tutto particolare (Burton, Gunther e Higley 1992). Non si può dire però che nella loro azione gli attori politici siano liberi da ogni vincolo. Il loro controllo sui fattori in gioco è basso e, soprattutto, sono limitate le loro risorse. In sintesi possiamo dire che mentre in una democrazia consolidata lo spazio politico può essere concepito come una costante, nelle nuove democrazie è invece una variabile.

Al livello della politica di massa il tasso di volatilità elettorale offre un primo indicatore del grado di strutturazione dello spazio politico. In uno spazio politico strutturato la volatilità dovrebbe essere bassa: le dimensioni dello spazio politico sono infatti ben definite; i partiti hanno una collocazione consolidata e ben conosciuta rispetto a queste e lo stesso vale per una parte cospicua degli elettori; la mobilità dei voti sarà quindi poco rilevante quantitativamente e sarà delimitata entro confini abbastanza modesti (Bartolini e Mair 1990). In uno spazio politico poco strutturato come quello delle nuove democrazie queste condizioni non si verificano (o si verificano solo in misura limitata), di conseguenza la mobilità dovrebbe essere assai più alta. In effetti questo è quanto si verifica nella maggior parte dei casi nel primo periodo dopo il ritorno alla democrazia (cfr. tab. 1) ${ }^{2}$. Gli alti indici di volatilità delle prime elezioni democratiche ${ }^{3}$ indicano che la strutturazione a livello elettorale dello spazio politico non è una acquisizione immediata.

Se dal piano elettorale ci spostiamo a quello degli attori partitici e consideriamo, da un lato, la loro dimensione organizzativa e, dall'altro, le interrelazioni tra di essi, possiamo utilizzare una serie di fenomeni - dalle scissioni alle fusioni di partiti, dalla creazione alla rottura di coalizioni - per valutare il proces-

2 Per una discussione più approfondita sul rapporto tra volatilità elettorale, strutturazione partitica e consolidamento democratico rinvio a Morlino (1986 e 1995).

${ }^{3}$ Per avere un metro comparativo si può ricordare che Bartolini e Mair (1990, 68 e 98-99) avevano calcolato una volatilità media dell' $8,6 \%$ su 303 elezioni europee relative agli anni $1885-1985$ e una media di circa il $10 \%$ per gli anni quaranta di questo secolo. 
ТАВ. 1. La volatilità elettorale dopo la ridemocratizzazione

\begin{tabular}{lccc}
\hline & \multicolumn{3}{c}{ Elezioni nazionali } \\
\cline { 2 - 4 } & Seconde & Terze & Quarte \\
\hline Austria & 12 & 4 & 6 \\
Francia & 6 & 20 & 20 \\
Germania & 21 & 9 & 11 \\
Italia & 23 & 14 & 5 \\
Grecia & 22 & 27 & 6 \\
Portogallo & 11 & 10 & 5 \\
Spagna & 11 & 42 & \\
Bulgaria & 20 & & \\
Polonia & $70 ?$ & $25+$ & \\
Rep. Ceca & $28+$ & & \\
Rep. Slovacca & $41+$ & & \\
Romania & $30+$ & & \\
Ungheria & 23 & & \\
\hline
\end{tabular}

Fonti: Bartolini e Mair (1990), Mackie e Rose (1991), e vari numeri di «Electoral Studies». Le elezioni prese in considerazione sono per l'Austria: 1949, 1953, 1956; per la Francia: 1946b, 1951, 1956 (in questo caso per la vicinanza temporale ho saltato dal computo le elezioni del 1945 ed ho quindi contato come seconde elezioni quelle del novembre 1946); per la Germania: 1953, 1957, 1961; per l'Italia: 1948, 1953, 1958; per la Grecia: 1977, 1981, 1985; per il Portogallo: 1976, 1979, 1980; per la Repubblica Ceca: 1992; per la Slovacchia: 1992; per la Polonia: 1991, 1993; per la Bulgaria: 1991; per la Romania: 1992; per l' Ungheria: 1994. Dove le trasformazioni dei partiti sono state più rilevanti le misure della volatilità possono essere discutibili: nei casi polacco, ceco, slovacco e rumeno i valori con il segno + sono stati calcolati per difetto. La volatilità delle seconde elezioni polacche ha un segno ? per richiamare l'attenzione sul carattere speciale delle prime elezioni rispetto alle quali è stata calcolata.

so di strutturazione dello spazio politico (Morlino 1986). Tutti questi fenomeni sono infatti strettamente legati a tale processo.

I primi due fenomeni - scissioni e fusioni - si collocano al confine tra il livello del sistema partitico e quello delle unità partitiche. Nel caso di una scissione quelle che prima erano relazioni «intra-partitiche» tra fazioni (livello partitico) diventano relazioni «inter-partitiche» tra unità partitiche indipendenti (livello del sistema partitico). L'opposto vale per le fusioni; le relazioni tra due o più partiti (livello sistemico) cedono il passo a problemi di coesistenza interna tra fazioni (livello partitico). Nei sistemi consolidati la linea di confine tra i due livelli è generalmente piuttosto chiara ed è facile distinguere tra fenomeni che attengono all'uno o all'altro livello. I fenomeni di spostamento, nell'uno o nell'altro senso, della linea di confine sono 
piuttosto rari perché confliggono con interessi molto radicati e comportano rischi piuttosto rilevanti per gli attori politici coinvolti. Nei sistemi partitici nuovi e poco consolidati questi fenomeni dovrebbero invece essere più frequenti. La distinzione tra livello partitico e sistemico non è ancora ben definita sia perché le identità (organizzative e ideologiche) delle unità partitiche sono ancora incerte che perché le caratteristiche dello spazio sistemico sono ancora molto fluide. Di conseguenza, è più facile che problemi interni ai partiti si traducano in problemi del sistema partitico e viceversa. Pertanto saranno più numerosi $\mathrm{i}$ casi di conflitti intrapartitici che si traducono in scissioni, di coalizioni che danno luogo ad una fusione di partiti, e le situazioni in cui non è chiaro se un gruppo politico sia veramente un partito o semplicemente una fazione.

Qualche parola in più merita la questione delle coalizioni. Sulla importanza che lo studio delle coalizioni ha acquistato nella scienza politica contemporanea non c'è bisogno di insistere a lungo. I tipi di coalizioni, la loro dimensione, composizione e durata, la distribuzione dei payoffs tra i componenti sono tutti elementi che ci forniscono informazioni essenziali sulle caratteristiche dello spazio politico e sulle dinamiche della vita politica (Budge e Keman 1990; Laver e Shofield 1990). Il punto da sottolineare è che in una democrazia consolidata le coalizioni sono numericamente limitate e abbastanza facili da prevedere. La configurazione ben definita dello spazio politico pone vincoli piuttosto precisi al processo coalizionale perché esclude un buon numero di coalizioni teoricamente possibili. Va aggiunto inoltre che un fattore importante di cambiamento delle coalizioni sono le variazioni nel peso elettorale dei partiti, ma poiché queste come abbiamo visto tendono ad essere limitate, prevale generalmente la stabilità delle formule.

Le coalizioni riflettono le caratteristiche principali dello spazio politico: il suo carattere unidimensionale o pluridimensionale, la sua ampiezza, cioè la distanza tra i partiti o polarizzazione (Sartori 1976), la collocazione dei singoli partiti in questo spazio e la loro Koalitionsfäbigkeit. Nei sistemi partitici di nuova data le coalizioni saranno meno vincolate e più volatili a causa della mancanza di uno spazio politico ben strutturato. $\mathrm{Ci}$ dovremmo aspettare che le formule coalizionali cambino più facilmente come conseguenza delle mutevoli condizioni di uno spazio politico in via di definizione, della ricerca da parte dei partiti di una collocazione più favorevole da cui condurre la 
competizione, delle più frequenti nascite e morti di unità partitiche (anche in conseguenza di scissioni e fusioni).

Nello studio dei processi formativi dei nuovi sistemi partitici sembra utile, dunque, analizzare insieme coalizioni, fusioni e scissioni partitiche. Questi fenomeni, molto più vicini tra loro in un sistema partitico in via di formazione che in uno già stabilizzato, ci forniscono informazioni essenziali per tener dietro ai cambiamenti in corso negli anni cruciali delle nuove democrazie. Costituiscono inoltre il punto di partenza obbligato per cercare di comprendere i fattori che contribuiscono a dar forma ai nuovi sistemi partitici (e al nuovo spazio politico) dopo la fine dei regimi non democratici. Che ruolo hanno le eredità del passato (tanto quelle derivanti dalle prime esperienze democratiche che dal regime autoritario), le diverse modalità della transizione alla democrazia, le nuove issues che emergono sulla scena con la normalizzazione della vita politica una volta instaurato il regime democratico (Cotta 1993 e 1994)?

Attraverso una analisi comparata delle recenti democratizzazioni dell'Europa centro-orientale e di quelle dell'Europa occidentale mi propongo di esaminare lo sviluppo parallelo dei nuovi sistemi partitici e le trasformazioni dello spazio politico nei primi anni dopo la caduta del regime non democratico. In particolare l'attenzione si concentrerà sul passaggio dalla fase di transizione a quella successiva. $\mathrm{Ci}$ sono infatti fondati motivi per attendersi che con questo passaggio la configurazione dello spazio politico subisca mutamenti significativi (con conseguenze di rilievo per i partiti e il sistema partitico). Il periodo della transizione è infatti dominato dai problemi dell'uscita dal vecchio regime e della creazione delle basi di consenso necessarie per l'installazione del nuovo regime democratico. Naturalmente non mancano anche altri problemi di natura sostanziale (ad esempio economici), ma essi tenderanno ad essere subordinati ai precedenti, altrimenti la instaurazione democratica sarebbe messa in forse. Però, una volta che la transizione ha raggiunto il suo obiettivo e un regime democratico è stato instaurato, è abbastanza naturale attendersi che quegli altri problemi appaiano sulla scena. L'ambiente politico all'interno del quale operano gli attori politici, e in specie i partiti, sarà probabilmente abbastanza diverso nelle due fasi ${ }^{4}$. E così pure saranno diversi i ri-

${ }^{4}$ Credo che si possa trovare una interessante corrispondenza, pur con riferimento 
schi e le opportunità, i vincoli e gli incentivi che entrano nei calcoli politici degli attori.

\section{La fase di transizione}

Date le condizioni del tutto speciali del periodo di transizione e il fatto che questo stadio coincide con la rottura del precedente monopolio potestativo e con la fase formativa dei nuovi movimenti politici, è abbastanza ragionevole aspettarsi un suo impatto piuttosto significativo sia sui caratteri dei singoli partiti, sia sulle relazioni che si stabiliscono tra di essi. Questo però significa anche che i partiti e le coalizioni nate in questo periodo e che hanno ottenuto un certo successo affondano le loro basi in una situazione intrinsecamente transitoria. L'opposizione al vecchio regime, la necessità di superare gli ostacoli frapposti al processo di democratizzazione e, in generale, di tenerlo sul binario giusto hanno in genere un ruolo molto importante nel promuovere e tenere insieme durante il periodo iniziale partiti o movimenti o coalizioni. E il ruolo di queste prime formazioni politiche è cruciale, nel corso dei delicatissimi e a volte drammatici mesi o anni iniziali del processo, per garantire il successo della transizione. $\mathrm{Ma}$, una volta che questo fine è stato raggiunto e nuovi problemi politici si affacciano, la coesione, il seguito popolare e il prestigio di quei partiti potranno essere messi in questione e magari evaporare piuttosto rapidamente. È possibile addirittura che quelli che prima erano fattori di forza si trasformino in fattori di debolezza.

Uno dei prodotti più tipici delle transizioni sono le grandi coalizioni di tutti i partiti che si oppongono al vecchio regime (o quantomeno della maggior parte di essi). I processi di ridemocratizzazione che sono seguiti alla seconda guerra mondiale offrono chiari esempi di queste «grandi coalizioni di transizio-

a contesti diversi, tra queste considerazioni e gli assunti specificati da Budge e Keman nella loro teoria del comportamento coalizionale dei partiti. Essi infatti assumono che, nel contesto di una democrazia stabilizzata, prevalga un ordine nelle preferenze dei partiti democratici, in base al quale al primo posto venga la difesa nei confronti delle minacce al sistema democratico e solo dopo quella degli interessi relativi al conflitto di classe o di altro tipo (1990, 32 ss.). Allo stesso modo, ma nel contesto della transizione, io assumo che la preoccupazione per la costruzione della democrazia prevalga sugli altri fini finché questo obiettivo non è stato raggiunto. 
ne». In effetti, con la eccezione della Germania Federale, si può ben dire che le grandi coalizioni anti-fasciste hanno dominato le transizioni nell'Europa postbellicas.

Il caso italiano è abbastanza emblematico. L'inizio della transizione alla democrazia, dopo la crisi del 25 luglio 1943 del regime fascista e la prima liberalizzazione della vita politica, vede i rinati partiti convergere rapidamente verso una formula di grande alleanza. Spinge in questa direzione, nonostante le passate divergenze ideologiche, una combinazione di fattori legati alla situazione: la necessità di combattere contro l'occupazione tedesca e il restaurato regime della Repubblica di Salò, l'esigenza di controllare meglio il processo di transizione e di spingere la monarchia sulla strada di una chiara democratizzazione (e non di una più limitata correzione del vecchio regime), ma anche la distribuzione delle risorse tra le forze politiche che fa sì che nessuna abbia la certezza di poter dominare da sola la transizione.

Una grande coalizione costituiva quindi per la maggior parte degli attori partitici una soluzione razionale in quel contesto. Le regole base dell'alleanza erano quelle della parità tra le forze politiche e della unanimità. Ma la condizione necessaria era che le questioni che potevano dividere i partiti fossero rinviate ad un momento successivo o spostate ad un'altra istanza decisionale. L'esempio più rilevante è quello della decisione sulla forma statale. Il dissenso tra monarchici e repubblicani può essere superato rinviando ad un referendum la scelta. Ma la mancanza di una omogeneità politica sostanziale all'interno della coalizione ciellenistica non avrebbe tardato a manifestarsi con il procedere del processo di transizione e con il cambiamento delle priorità dell'agenda politica (dalla questione dell'opposizione alle forze di occupazione e del vecchio regime ai problemi concreti della nuova democrazia). Non a caso già nel 1946 si avrà la defezione sulla destra del Partito Liberale e nel 1947 si delineerà la frattura sul versante sinistro.

Il caso francese presenta notevoli somiglianze con quello italiano. Anche qui l'inizio è nel segno della grande coalizione anti-fascista. I primi governi sono chiaramente la continuazione

${ }^{5}$ Mi limito qui a discutere i casi austriaco, francese e italiano; lascio invece da parte le brevi ridemocratizzazioni dell'Europa centro-orientale. Le speciali condizioni nelle quali si sono sviluppate (l'occupazione sovietica) hanno lasciato agli attori politici locali margini di libertà molto più ridotti e ne hanno determinato la rapida fine. 
delle alleanze nate nella lotta contro l'occupazione tedesca (non a caso sono guidati appunto dal leader della resistenza, de Gaulle) e includono tutti i partiti principali non coinvolti nel crollo del regime di Vichy. Ma ancor più rapidamente che in Italia si manifestano le crepe nella coalizione. Qui i primi motivi del contendere riguardano le scelte costituzionali. La presenza di un leader di grande peso non riconducibile ai partiti della coalizione e con una propria ben definita visione degli assetti istituzionali (e in specie del ruolo del capo dello stato) e, in aggiunta, la scelta di sottoporre i progetti costituzionali a ratifica referendaria, con l'effetto di invogliare i partiti ad adottare atteggiamenti intransigenti in sede costituente in attesa di una rivincita nell'arena popolare, sono forse $\mathrm{i}$ due fattori che maggiormente accelerano la disgregazione. Già all'inizio del 1946 la grande coalizione perde il suo leader, il quale si ritira ai margini della vita politica (Williams 1958, 14 ss.). Nel 1947 seguirà poi la rottura tra il Partito Comunista e gli altri partner; l'occasione sono dissensi su questioni di politica interna (le politiche salariali) ed internazionale (la decolonizzazione in Indocina); ma sullo sfondo aleggia la grande contrapposizione Est-Ovest. Questa rottura, che sarà bilanciata dall'inclusione dei partiti di destra (prima tenuti ai margini per il loro coinvolgimento nel regime di Vichy), segna a tutti gli effetti la fine della grande coalizione di transizione.

Tra i casi postbellici il terzo, quello austriaco, ha un avvio simile agli altri due ma poi segue una evoluzione diversa. Anche qui si forma inizialmente una coalizione di tutti i partiti autorizzati all'attività politica nelle condizioni di occupazione (che si prolungano in Austria fino al trattato del 1955). Il primo governo provvisorio, guidato dal leader socialista Renner, comprende infatti su basi grosso modo di parità Socialisti, Cattolici e Comunisti. La coalizione prosegue anche dopo le prime elezioni (novembre 1945) e fino al novembre 1947 quando il Partito Comunista come nei casi francese e italiano (ma qui si tratta di un partito molto più piccolo) viene escluso dal governo. La specificità austriaca sta nel fatto che la grande coalizione dei due partiti maggiori, SPÖ e ÖVP non si scioglie ma continua fino al 1966 (dopo di che sarà seguita da 17 anni di governi monopartito). L'Austria è l'unico caso nel quale l'ampia coalizione formatasi nella fase della transizione (o per essere più precisi il grosso di questa) sopravviverà a lungo a quel periodo. Ci si può chiedere naturalmente se la grande coalizione austria- 
ca sia stata prodotta effettivamente dalle condizioni specifiche della transizione. La risposta mi pare debba essere positiva almeno per la sua fase iniziale. In effetti la scelta di questo tipo di coalizione anche quando in teoria sarebbero state possibili altre soluzioni, come un governo monopartitico (ad esempio dopo le elezioni del 1945) o una piccola coalizione (con i Liberali dopo il 1949) rispondeva a tre specifici problemi connessi alla transizione alla democrazia. Il primo era la necessità di avere un governo autorevole per far fronte all'occupazione militare seguita alla guerra e riguadagnare al più presto la sovranità nazionale. Il secondo era quello di superare nella nuova democrazia la ostilità tra $\mathrm{i}$ due schieramenti politici principali che aveva portato alla rovina la prima repubblica ${ }^{6}$. Il terzo era la esclusione della terza forza politica - il Partito Liberale - che, per la sua collusione con il regime nazista e il suo nazionalismo pangermanico, risultava difficilmente compatibile con i principi della nuova democrazia e della indipendenza nazionale ${ }^{7}$.

La durata della grande coalizione ben al di là del periodo di transizione sembra indicare che forse alcuni di questi fattori non avevano carattere solo temporaneo e legato ai problemi di quella fase ma una portata di più lungo termine. L'alto grado di continuità nella vita politica austriaca di questo secolo offre probabilmente la chiave per capire questa peculiarità. La capacità dei due partiti principali della prima repubblica di sopravvivere come $\mathrm{i}$ protagonisti anche della seconda li poneva di fronte ad un serio dilemma: scegliere la strada della aperta competizione con il rischio di ridare esca alle antiche ostilità che avevano fatto naufragare la prima democrazia, oppure ridurre drasticamente il tasso di competizione attraverso un accordo stabile che evitasse il rischio di ripetere un'esperienza dalla quale in definitiva entrambe le parti erano state seriamente danneggiate. Le memorie negative del passato e $\mathrm{i}$ problemi del presente hanno certo contribuito a far pendere la bilancia verso la cooperazione. Tuttavia il «problema austriaco» non poteva essere considerato solo un problema della fase di transizio-

\footnotetext{
${ }^{6}$ Per una più ampia discussione di come si passi dalla ostilità tra i grandi partiti alla cooperazione postbellica si vedano i classici studi di Wandruska (1954), Engelmann (1966) e Bluhm (1973).

7 Occorre ricordare che il nazionalismo austriaco tra le due guerre aveva avuto come peculiarità di essere pantedesco ed aveva puntato alla Anschluss con la Germania in nome appunto della comune tradizione germanica.
} 
ne. Non aveva cioè soltanto a che fare con la questione dell'instaurazione della democrazia, bensì con il problema di più lungo periodo di assicurarne il consolidamento. La strategia della cooperazione tra i due partiti principali poteva quindi essere vista come una risposta (necessariamente prolungata nel tempo) al possibile risorgere di pericolose tensioni ${ }^{8}$. Va aggiunto che anche il deficit di Koalitionsfäbigkeit del Partito Liberale era un fattore a effetti durevoli.

Se dal livello delle relazioni tra i partiti (come è il caso quando si parla di coalizioni) ci spostiamo a quello delle unità partitiche possiamo trovare altri fenomeni strettamente legati alle specifiche condizioni della transizione. Non è raro infatti che in questo periodo assumano una importanza di particolare rilievo partiti o movimenti politici che si definiscono essenzialmente sulla base dell'opposizione al vecchio regime, oppure nascono come strumenti politici quasi ad boc per far fronte ai problemi speciali della transizione alla democrazia. Da questo punto di vista si pongono sulla stessa linea delle grandi coalizioni. Solidarnosc in Polonia, i due movimenti gemelli - Foro Civico e Pubblico contro la Violenza - in Cecoslovacchia, ma anche, per quanto a prima vista possa sembrare un po' forzato, la UCD in Spagna offrono interessanti esperienze in proposito. Tutte e tre queste realtà politiche nascono in stretto collegamento con i processi di transizione alla democrazia. E, dovremmo aggiungere, le loro differenze hanno a che fare proprio con le peculiarità delle rispettive transizioni. Inoltre, in maniera assai simile a quella delle «coalizioni di transizione» di Francia e Italia, queste tre forze politiche, dopo la fase gloriosa e coronata di successo della transizione, attraversano una crisi profonda quando questa si è compiuta. La UCD praticamente sparisce lasciando dietro di sé solo delle schegge partitiche che si riveleranno incapaci di pesare nel gioco politico. Solidarnosc esplode dando luogo a una serie di partiti con profili politici e ideologici diversificati. Quanto al Foro Civico e al Pubblico contro la Violenza, subiscono entrambi gravi fratture: del primo sopravvivono, dopo essersi trasformate, alcune componenti; il secondo praticamente sparisce dalla scena. Come vedremo più in detta-

\footnotetext{
${ }^{8}$ Conviene ricordare che nella prima repubblica la cooperazione tra Cattolici e Socialisti era stata di breve durata e aveva presto lasciato il posto ad una ostilità reciproca sempre più forte (Wandruska 1954).
} 
glio si possono trovare parallelismi molto significativi con i primi tre casi discussi. Il caso polacco e quello cecoslovacco presentano interessanti somiglianze con quelli francese e italiano. Come nei due casi occidentali anche qui l'opposizione contro il vecchio regime è il fattore dominante e unificante durante la transizione. Ma nei due paesi dell'Europa centrale questo produce la nascita di un ampio movimento politico (Solidarnosc in Polonia e OF-VPN in Cecoslovacchia) invece di una grande coalizione dei partiti democratici. Le ragioni della differenza non sono difficili da spiegare: da un lato c'è la estrema debolezza, dopo quarant'anni di regime comunista, di una tradizione politica dalla quale possano rinascere partiti democratici di qualche consistenza (Cotta 1993 e 1994), dall'altro c'è un forte rigetto dell'idea stessa di partito dopo un regime non democratico fortemente centrato sul partito unico. Mancando dunque le componenti partitiche per una grande coalizione, il primo passo è quello di una mobilitazione «non partitica» in opposizione al regime comunista. Il carattere composito (potremmo dire coalizionale) di questi movimenti di opposizione è comunque chiaro sin dall'inizio. In effetti il loro successo deriva proprio dall'essere riusciti a far convergere filoni diversi di opposizione al regime che in precedenza avevano marciato in ordine sparso. Questo è particolarmente chiaro nel caso di Solidarnosc che, a differenza dei movimenti di opposizione al regime comunista degli anni precedenti, era riuscita alla sua apparizione nel 1980 a superare le precedenti divisioni tra movimenti di opposizione cattolici e laici, intellettuali e operai e anche nazionalisti e liberali (Grilli di Cortona 1989). La vicenda del Foro Civico e del Pubblico contro la Violenza è stata più breve, ma anche in questo caso si può facilmente individuare una «coalizione» di orientamenti diversi uniti dall'opposizione al regime al potere.

Entrambi i movimenti hanno avuto un ruolo centrale nello spingere il regime comunista sulla strada della democratizzazione e, infine, alla sconfitta ed entrambi hanno vinto le prime elezioni libere o semi-libere (come quelle polacche del 1989) ottenendo così la guida dei primi governi democratici. Ma la vittoria ha segnato anche l'inizio del loro declino. Rivalità tra leader e dissensi ideologici hanno cominciato presto a minare l'unità dei due movimenti.

Dopo una prima grave spaccatura già alle elezioni presidenziali del 1990, Solidarnosc giunge ormai divisa in molteplici frammenti alle prime elezioni pienamente competitive (quelle 
del 1991). Sei o sette di questi saranno tra gli attori rilevanti del gioco politico fino alle elezioni del 1993. Le loro identità politiche e le relazioni tra di essi saranno definite dai nuovi problemi che emergono nel dopo-transizione. Naturalmente alcuni dei problemi derivanti dalle conseguenze del vecchio regime (riforma economica, privatizzazioni, leggi di epurazione) hanno in questa fase un peso notevole. Questo fatto, al quale si è aggiunta l'esistenza di partiti eredi delle formazioni politiche del vecchio regime (segnatamente la Alleanza Democratica di Sinistra, erede del Partito Comunista, e il Partito Contadino, continuazione dell'omonimo partito del periodo precedente), ha certamente contribuito a mantenere in vita anche nel nuovo sistema partitico una linea di divisione che oppone i partiti eredi di Solidarnosc a quelli derivati dal regime comunista. Questa linea, che interseca le altre linee di divisione, ha posto ancora dei vincoli significativi ai processi di formazione delle coalizioni tra il 1991 e il 1993. Ciononostante, alcuni tentativi di superare questa linea di divisione per poter costruire maggioranze capaci di risolvere i nuovi problemi del paese si sono manifestati già in questo periodo. In particolare si può notare che il «meno pericoloso» dei due partiti post-comunisti, cioè il Partito Contadino Polacco (PSL) ha acquisito via via un grado di accettabilità crescente. Ad un certo punto ha contribuito a sostenere il governo Olszewski contro una mozione di censura del Sejm mentre in seguito il suo leader Pawlak si è prestato ad un tentativo presidenziale di mettere in piedi un governo più amico dopo la caduta di Olszewski. Ma anche l'Alleanza Democratica di Sinistra, durante i governi Bielecki e Suchocka, ha di tanto in tanto fornito il sostegno parlamentare alla maggioranza su alcune questioni legislative. Infine, con le elezioni del 1993 è apparso chiaro che la linea di divisione tra forze eredi di Solidarnosc e partiti post-comunisti può certamente avere ancora un peso rilevante ai fini della definizione degli allineamenti elettorali e degli accordi coalizionali, ma ha assunto un significato ben diverso dallo scontro tra Solidarnosc e il regime comunista di appena pochi anni prima. In discussione sono ora le politiche sostanziali e gli interessi sociali toccati da queste; non più invece la questione del vecchio regime. I tempi della coalizione anti-regime e della frattura insormontabile tra «loro» e «noi» sono finiti.

Nel caso cecoslovacco - ma dal 1992 bisogna ovviamente parlare di due casi distinti, quello ceco e quello slovacco - la trasformazione si presenta con caratteristiche un po' diverse. 
Entrambe le ali del movimento di opposizione, poco dopo aver ottenuto il potere grazie proprio alla loro identità di forze opposte al vecchio regime, subiscono scissioni dalle quali emergeranno i partiti principali dei due nuovi stati nati dalla rottura della federazione. Nella repubblica ceca nasce il Partito Civico Democratico di V. Klaus, in quella slovacca lo HZDS di Meciar. Anche se si può dire che entrambi i partiti (ma ciò vale con più chiarezza nel caso del partito di Klaus che di quello di Meciar) sono nati dai movimenti di opposizione, tutti e due segnano un netto distacco dai problemi della transizione e il passaggio alle tematiche del dopo-transizione. Il Partito Civico Democratico fonda la sua identità essenzialmente sulla politica economica; viceversa lo HZDS di Meciar pone al centro della sua strategia politica la questione dell'indipendenza slovacca. In entrambi i casi gli altri partiti nati dalla scissione dei due fronti non hanno raggiunto un peso molto significativo.

Nei due paesi del centro Europa, in maniera dunque abbastanza simile ai casi italiano e francese, si può rilevare da un lato il ruolo importante giocato dalle formazioni politiche (movimenti nei primi due casi, coalizioni negli altri) nate dalla lotta al vecchio regime, dall'altro la loro rapida crisi. In Polonia, e qui il parallelismo con il caso francese è singolare, la crisi della formazione politica di transizione è stata accelerata dal ruolo ingombrante del leader carismatico. Walesa, come in Francia de Gaulle, non trova dopo la restaurazione della democrazia un posto soddisfacente nelle forze politiche esistenti e nel sistema istituzionale del nuovo regime. La peculiarità dei due casi centroeuropei rispetto a quelli dell'Europa occidentale sta invece nel forte «ritorno» dei partiti successori del regime non democratico secondo quella che ormai (con la eccezione della repubblica ceca) sembra una caratteristica comune dei sistemi politici post-sovietici. Ai nostri fini il punto da rilevare è che ciò comporta, almeno a prima vista, una certa continuità tra periodo della transizione e periodo successivo. Sembra infatti che i due schieramenti rimangano gli stessi nei due periodi. Ad un esame più attento risulta invece chiaro che la continuità è solo apparente perché l'ambiente sistemico e l'oggetto della competizione politica sono profondamente cambiati. Non a caso, infatti, nella nuova fase si rivela molto più difficile rimettere insieme il vecchio fronte anti-comunista.

Tra i casi dell'Europa occidentale quello spagnolo merita un'attenzione speciale per la vicenda abbastanza clamorosa del- 
l'UCD. È interessante intanto soffermarsi sul fatto che nella transizione spagnola nonostante i suoi caratteri marcatamente consensuali (o forse proprio in forza di questi?) (Gunther 1992), non si è sviluppata una vera e propria coalizione dei partiti democratici. La UCD, grazie al ruolo cruciale svolto dal suo leader Suárez durante la transizione e al grande successo nelle prime elezioni libere (1977), ha potuto infatti condurre da sola il gioco di governo. Dato il credito acquistato da questo partito per la transizione indolore alla democrazia, la sua disastrosa sconfitta alle elezioni del 1982 e la sua fine come partito rilevante potrebbero apparire inspiegabili, a meno che non si tengano in conto, da un lato, le caratteristiche specifiche di questo partito (Huneeus 1985) e, dall'altro, le trasformazioni dello spazio politico intervenute nel corso di questi anni. In particolare occorre innanzitutto ricordare che la UCD nasce come una confederazione elettorale tra molti partiti (o proto-partiti) caratterizzati da identità politiche differenti. In secondo luogo, che l'originario fattore unificante è strettamente collegato alle problematiche della transizione. Si trattava essenzialmente della esigenza di costruire una coalizione di centro che riunisse la opposizione moderata al vecchio regime e i settori più aperti dell'élite franchista in modo da contenere il rischio di un ritorno a quello scontro tra destra conservatrice e sinistra radicale che aveva portato alla rovina la seconda repubblica (Gunther, Sani e Shabad 1988, 92 ss.). In un certo senso si potrebbe dire che la UCD incarna al suo interno quella esigenza di una larga coalizione che è un elemento così frequente nelle transizioni di regime. Non si tratta però tanto di una coalizione «contro» il vecchio regime quanto di una coalizione per superare la frattura tra vecchio e nuovo unendo i moderati dei due campi (e quindi separandoli dalle estreme di ciascuno dei due). Le ragioni di questo tipo speciale di coalizione possono essere rintracciate nelle vicende specifiche del passato politico spagnolo. Se il franchismo aveva significato la vittoria di uno dei due schieramenti della guerra civile, la democrazia non poteva significare invece la vittoria, dopo quarant'anni, dell'altra metà della Spagna. Doveva invece essere fondata su un accordo tra i due fronti, cioè proprio quello che era mancato negli anni trenta. Nella prima fase della transizione, quando non era ancora chiaro se $\mathrm{i}$ «duri» del vecchio regime, da un lato, e le forze più intransigenti dell'opposizione, dall'altro, sarebbero state disposte a giocare in maniera cooperativa il gioco della ridemocratizzazione 
(Gunther 1992), una coalizione capace di collocarsi al centro dello spettro politico deve essere apparsa a molti leader politici, pur di diverso orientamento, la strada più responsabile e con maggiori possibilità di successo.

In questo senso il caso spagnolo presenta alcune somiglianze con quello austriaco. In entrambi i casi lo scontro tra i due fronti della sinistra e della destra durante la prima esperienza democratica era degenerato in una guerra civile (certo molto più lunga e sanguinosa in Spagna), seguita dalla vittoria di una delle due parti che aveva affossato la democrazia. Il superamento del passato doveva quindi essere basato sulla possibilità di sanare questa frattura. In un paese come l'Austria nel quale le espressioni politiche dei due campi avversi, cioè il Partito Socialista e quello Cattolico, era state capaci, grazie alla loro tradizionale forza organizzativa, di sopravvivere senza troppi danni al periodo autoritario, una loro coalizione rappresentava la soluzione più ovvia. In Spagna la tradizione partitica molto più debole, in specie al centro e alla destra dello schieramento partitico, e la maggiore durata della interruzione autoritaria rendevano più difficile una soluzione analoga a quella austriaca. La creazione di un partito centrale rispetto allo spettro politico offriva dunque una «alternativa funzionale» più semplice per risolvere il problema cruciale della transizione. D'altra parte, però, la persistenza del partito stesso (e non soltanto di un accordo coalizionale) era a rischio se le condizioni che ne avevano favorito la nascita si fossero trovate a cambiare. Ciò è appunto quanto è accaduto in Spagna dopo non molto tempo. La sconfitta dell'ala intransigente del franchismo e la linea moderata adottata dalla sinistra hanno creato assai presto una situazione nella quale i rischi del ripetersi di uno scontro tra sinistra e destra si sono drasticamente ridotti. Le ragioni che avevano sostenuto la nascita dell'UCD hanno perso così gran parte della loro forza, mentre hanno potuto avere il sopravvento le forze centrifughe interne (tensioni organizzative di un partito-federazione, rivalità personali tra $\mathrm{i}$ leader delle diverse fazioni, dissensi ideologici tra ala confessionale e laica, tra ex-franchisti e oppositori del vecchio regime) (Huneeus 1985).

L'elemento che accomuna i tre paesi è che i partiti o movimenti politici che avevano avuto un ruolo centrale nella transizione sono poi scomparsi o si sono trasformati in nuovi partiti profondamente diversi. Una delle ragioni di ciò sta nel fatto che questi partiti o movimenti erano in un certo senso delle «fede- 
razioni» eterogenee che raggruppavano sotto uno stesso tetto orientamenti ideologici (e leader) assai diversi e uniti essenzialmente dalla necessità di combattere il vecchio regime (è il caso di Solidarnosc e dell'OF/VPN) o di condurre a buon termine una transizione pacifica (UCD). Nel momento in cui si sono trovati a dover fronteggiare i nuovi problemi emergenti dopo il completamento della transizione, le differenze ideologiche e le rivalità tra leader non hanno potuto più essere superate e l'unità si è infranta. Parallelamente alla crisi di queste forze politiche anche la struttura del sistema partitico si è profondamente rimodellata.

Tra i paesi dell'Europa centro-orientale due casi, quelli della Bulgaria e della Romania, presentano alcune importanti peculiarità rispetto alle esperienze sin qui esaminate. E vero che anche in questi casi si sono formate durante la transizione larghe coalizioni di partiti in opposizione al vecchio regime. Questo è avvenuto prima in Bulgaria, dove la Unione delle Forze Democratiche ha raccolto sotto le sue insegne una varietà di partiti e movimenti allo scopo di sfidare il Partito Comunista che, riciclatosi sotto l'etichetta di Partito Socialista Bulgaro, si dimostrava capace di conservare un seguito popolare non trascurabile. In Romania la Convenzione Democratica Romena si è formata solo dopo le prime elezioni che avevano visto la sconfitta dei partiti anticomunisti e la vittoria del Fronte di Salvezza Nazionale di Iliescu. Ciò che rende questi casi differenti, almeno nella fase iniziale, rispetto ai precedenti è la capacità del partito legato più o meno direttamente al vecchio regime comunista di sopravvivere come uno degli attori principali durante la transizione. In Bulgaria, addirittura, il partito-guida del vecchio regime sopravvive come forza politica di primo rango alle prime due tornate di elezioni democratiche cambiando poco più che il nome (Szajkowski 1991). È abbastanza comprensibile allora che anche la coalizione anti-comunista sia sopravvissuta per un periodo relativamente più lungo che in altri paesi dell'Europa centro-orientale. Il caso romeno è invece meno chiaro. Il primo punto da rilevare è che il Fronte di Salvezza Nazionale è, all'inizio, una forza politica molto più ambigua del Partito Socialista Bulgaro. Per valutare questo partito occorre tener conto del suo ruolo tanto di attore cruciale nella distruzione del regime personale di Ceausescu, quanto di strumento capace di assicurare la sopravvivenza di una parte almeno del vecchio establishment politico. Il fatto che un fronte opposto al vecchio 
regime non si sia sviluppato tempestivamente è probabilmente dipeso proprio da questa ambiguità. Solo più avanti, quando è apparsa più chiara la continuità del FSN con il passato, si è potuta formare una contro-coalizione (la Convenzione Democratica Romena). È interessante notare come questa ambiguità abbia coinvolto anche uno dei vecchi partiti di opposizione, il Partito Liberale Nazionale, portandolo ad associarsi per qualche tempo tanto al governo Stoloyan basato sul FSN che al fronte di opposizione.

In questi due paesi il confronto tra vecchio regime e forze di opposizione si mantiene dunque in vita più a lungo anche dopo che è stata avviata la fase della politica competitiva. La ragione di questa peculiarità sta fondamentalmente nella forte dose di continuità tra vecchio e nuovo durante la transizione. Piuttosto che addentrarci qui in un tentativo di spiegazione di questa continuità (ci si potrebbe rifare forse al grado maggiore di consenso popolare di cui questi regimi comunisti godevano rispetto agli altri, o anche alla maggiore debolezza delle forze di opposizione) sembra più rilevante chiedersi se questa peculiarità sia destinata a rimanere tale a più lungo termine. Questo interrogativo potrebbe tradursi in un altro: rispetto agli altri paesi dell'Europa centro-orientale Bulgaria e Romania devono essere considerati due casi di sviluppo ritardato (perché non c'è stata una rottura netta con il vecchio regime) o, al contrario, hanno addirittura anticipato un trend comune poi a gran parte di quest'area (cioè il ritorno in forze dei partiti post-comunisti)? Una risposta a questi interrogativi richiederebbe di verificare se la contrapposizione di regime sia rimasta in questi paesi l'asse principale dello spazio politico oppure se il suo posto sia stato preso da altre dimensioni di conflitto. Sul punto torneremo più avanti.

Alla fine di questa prima parte dell'analisi sono rimasti fuori tre paesi - Germania, Grecia e Ungheria - che si presentano come eccezioni. In questi paesi, infatti, il periodo della transizione sembra avere avuto un impatto molto minore sulla conformazione del sistema partitico. Né grandi coalizioni né partiti o movimenti politici orientati specificamente alla soluzione dei problemi della estricazione dal vecchio regime sembrano aver avuto gran peso.

Il caso tedesco è emblematico: dopo la caduta del regime nazista la vita politica nella Germania occidentale ha seguito la via di una piena competizione tra la CDU/CSU con i suoi allea- 
ti da una parte e la SPD dall'altra (Pridham 1977 e Smith 1979). A livello nazionale non si è mai costituita una grande coalizione dei partiti anti-nazisti'. Ha prevalso subito il cleavage «naturale» tra i grandi partiti democratici, che ha quindi strutturato in maniera durevole lo spazio politico.

In un contesto storico molto differente il caso greco ha seguito una linea di sviluppo non troppo dissimile (quantomeno rispetto a questo specifico punto). Una forte competizione tra $i$ partiti ha prevalso. Il leader della destra democratica Karamanlis, insieme al suo partito, ha preso sin dall'inizio il pieno controllo del governo e del processo di democratizzazione (Diamandouros 1986). La scelta di lasciar cadere la possibilità di una coalizione con gli oppositori di sinistra della dittatura e di basare i primi governi su una destra rinnovata in senso democratico è stata probabilmente dettata dalla necessità di evitare il rinascere di una destra non democratica che altrimenti avrebbe più facilmente potuto sfruttare le vecchie paure nei confronti delle sinistre e usare l'argomento propagandistico del governo «dominato dalle sinistre» per attaccare Karamanlis stesso. In ogni caso, quali che siano state le ragioni di Karamanlis, gli sviluppi degli anni successivi hanno mostrato l'efficacia di questa strategia nel consolidare il sistema partitico ben al di là della fase di transizione. A differenza della UCD di Suárez, la Nuova Democrazia di Karamanlis è stata in grado di sopravvivere al «ritorno alla normalità», di superare ripetute sconfitte elettorali e di riacquistare il potere dopo un periodo all'opposizione. Un po' come in Germania la prima strutturazione del sistema partitico si è rivelata capace di fondarne gli sviluppi anche più a lungo termine.

Nell'ambito dell'Europa post-comunista il caso ungherese sembra, almeno all'inizio, quasi una replica di questo modello. A differenza che negli altri paesi di quest'area un fronte unito delle opposizioni non ha avuto gran peso durante la transizione. Le forze di opposizione si sono divise piuttosto precocemente in due gruppi principali (il momento si può far coincidere con la prima scelta istituzionale della transizione, quella relativa al metodo di elezione del capo dello stato) (Agh 1992). E, addirittura, nelle prime elezioni libere la competizione tra le di-

\footnotetext{
9 Va osservato tuttavia che a livello di Land ci sono stati spesso tra il 1946 e il 1948 governi basati su larghe coalizioni (Ruhl 1982, 497-500).
} 
verse forze politiche anti-comuniste (il Foro Democratico Ungherese con i suoi alleati da una parte, l'Alleanza Liberaldemocratica e l'Alleanza dei Giovani Democratici dall'altra) ha praticamente relegato in secondo piano la più ovvia contrapposizione tra partiti anti-comunisti e post-comunisti.

Ho finora lasciato da parte il caso portoghese per la difficoltà a collocarlo tra gli altri. In effetti i suoi sviluppi sono stati assai peculiari. La spinta unificante tra le forze politiche prodotta inizialmente dall'euforia rivoluzionaria è stata rapidamente interrotta dai drammatici conflitti insorti tra queste stesse forze sul controllo e la direzione del processo di transizione. In effetti si potrebbe dire che il successo iniziale del Partito Socialista sia stato dovuto in larga misura alla sua capacità di presentarsi, un po' come la UCD in Spagna, come una soluzione intermedia tra le due posizioni estreme del conflitto. E il suo successivo declino potrebbe essere considerato la conseguenza dell'attenuarsi di quello stesso conflitto. Si potrebbe quindi dire che il PSP ha costituito il centro della prima strutturazione del sistema partitico portoghese, dominata dai problemi della transizione. Certo è che la strada del consolidamento del sistema partitico si rivelerà abbastanza lunga e complicata, in parte per il permanere del conflitto sulle soluzioni istituzionali (in particolare la questione dei poteri del capo dello stato), in parte per le difficoltà incontrate nei primi anni dall'area di centro-destra nel darsi un asssetto partitico stabile.

Da questa rapida discussione dei casi risulta dunque che la apparizione di larghe coalizioni o di ampi movimenti (conglomerati di una varietà di gruppi) che basano in misura significativa la propria identità politica sulle esigenze speciali della transizione alla democrazia è un fenomeno piuttosto comune (vedi tab. 2). Queste formazioni politiche orientate alla transizione (sia che si tratti di coalizioni che di fronti unitari) sono, da un lato, il risultato di una lotta comune contro il vecchio regime, dall'altro, la risposta alle sfide e ai problemi della transizione. In particolare $\mathrm{i}$ loro sforzi sono orientati verso una $\mathrm{o}$ più $\mathrm{di}$ queste finalità: 1. spingere il vecchio regime verso la (piena) democratizzazione (Cecoslovacchia, Polonia, Bulgaria); 2. contendere il controllo sul processo di transizione ad altri attori interni e/o esterni (Austria, Italia, Romania); 3. superare le eredità negative della prima democrazia, in particolare rivalità radicali tra partiti (Austria e Spagna); 4. realizzare un equilibrio tra le diverse forze di opposizione (Francia, Italia, Polonia). 
TAB. 2. Variazioni nella strutturazione iniziale della vita politica

\begin{tabular}{lllll}
\hline $\begin{array}{l}\text { Coalizioni o movimenti di opposizione } \\
\text { al vecchio regime }\end{array}$ & & \multicolumn{2}{c}{$\begin{array}{c}\text { Coalizioni o partiti finalizzati al } \\
\text { superamento delle divisioni del passato }\end{array}$} \\
\cline { 5 - 6 } coalizioni & movimenti & & coalizioni & partiti \\
\cline { 4 - 5 } $\begin{array}{l}\text { Francia } \\
\text { Italia }\end{array}$ & $\begin{array}{l}\text { Cecoslovacchia } \\
\text { Polonia }\end{array}$ & & Austria & Spagna \\
\hline
\end{tabular}

Equilibrio tra formazioni politiche del vecchio regime e formazioni di opposizione

Competizione tra partiti democratici

Bulgaria

Germania

Romania

Grecia

Ungheria

(Portogallo)

La «politica di transizione» assume più facilmente la forma della grande coalizione quando una pluralità di partiti con una propria identità ideologica e organizzativa riescono a formarsi precocemente appena comincia a manifestarsi la crisi del regime non democratico. Questo è in genere tanto più facile quanto più le tradizioni politiche democratiche sono state forti (Cotta 1993 e 1994). Quando non si realizzano queste condizioni è più facile che nascano ampi fronti di opposizione (Solidarnosc e il Foro Civico/Pubblico contro la Violenza ne sono gli esempi emblematici). Un caso in certo senso intermedio è quello di partiti (come la UCD in Spagna) che nascono dalla fusione di altri partiti (o pre-partiti), oppure di alleanze elettorali nelle quali i partiti aderenti mantengono in qualche misura la propria identità ma perdono gran parte della loro libertà di azione (è il caso della Unione delle Forze Democratiche in Bulgaria).

Dal momento che queste coalizioni, alleanze di partiti o movimenti sono una risposta ai comuni problemi della transizione, simili dovrebbero essere anche le loro difficoltà quando questi problemi saranno superati. La conseguenza dovrebbe essere che le coalizioni si scioglieranno (in tutto o in parte) e $i$ partiti intraprenderanno strade diverse (magari entrando anche in nuove coalizioni). Quanto ai movimenti di opposizione o conglomerati di partiti, la necessità di ridefinire la propria identità porterà a scissioni o addirittura a un completo tracollo. In conclusione si potrebbe dire che quanto più profondamente il processo di transizione con i suoi specifici problemi ha determi- 
nato i caratteri originari del sistema partitico, tanto più profonda è destinata ad essere anche la sua ristrutturazione dopo la fine di questa fase.

Come abbiamo visto ci sono anche alcune importanti eccezioni a questa regola. In alcuni paesi (Germania, Grecia, Ungheria) la transizione alla democrazia ha avuto un impatto meno specifico sulle realtà partitiche. In particolare non si sono sviluppati né grandi coalizioni né movimenti ad boc. Le ragioni sembrano essere state due: (a) per motivi interni od esterni la transizione alla democrazia e il raggiungimento dei suoi fini sono apparsi presto assicurati e comunque non tali da dover richiedere un'azione speciale da parte dei partiti ${ }^{10} ;(b)$ un gruppo politico ha individuato la possibilità di guidare da solo il gioco della transizione senza dover chiedere il sostegno degli altri e, contemporaneamente, il rischio di antagonizzare seriamente questi ultimi è sembrato ridotto.

\section{La ristrutturazione dello spazio politico dopo la transizione}

Una volta che le sfide e i problemi della fase di transizione hanno perso di importanza in quali direzioni si sviluppano i sistemi partitici e quali fattori fanno sentire la loro influenza? Ovviamente, se si vuole tentare di rispondere a questi interrogativi, i casi dell'Europa occidentale offrono all'osservatore sviluppi temporalmente più lunghi che consentono di valutare meglio la fase post-transizione. Nell'Europa centro-orientale disponiamo invece di periodi di osservazione ben più ridotti che non permettono di andare molto al di là di congetture.

In linea di massima la lezione che ricaviamo dalle esperienze europee occidentali è che gli sviluppi politici susseguenti alla fase di transizione sono influenzati dagli effetti combinati di cleavages di lungo periodo e di nuovi problemi e temi emergenti nella fase della restaurazione democratica. Il ritorno alla «normalità», dopo che i problemi della transizione sono stati risolti o hanno comunque perso la loro iniziale intensità, significa nella maggior parte dei casi che alcuni conflitti socio-politici

${ }^{10}$ In Germania perché la transizione era controllata e assicurata dalle potenze occupanti; in Ungheria perché è stata facilmente concessa dal partito unico; in Grecia perché i militari erano ormai in cerca di una via d'uscita dal cul de sac nel quale si erano infilati. 
«permanenti», o quantomeno di lunga durata (dai conflitti centro-periferia a quelli di classe, ecc.), ritornano sul proscenio dopo esserne stati temporaneamente scacciati dalla turbolenza della trasformazione di regime. Bisogna ricordare che erano stati in genere proprio questi conflitti che, non risolti efficacemente dalla prima democrazia, avevano creato il terreno favorevole per l'avvento del regime non democratico. Per lo più, tuttavia, nemmeno questo regime vi aveva dato una risposta soddisfacente nel lungo periodo ( $\mathrm{e}$ aveva finito a volte per trovare proprio in essi un fattore di crisi). Anche la nuova democrazia presto si trova a doverli riaffrontare ed essi forniranno alcune importanti basi per la strutturazione del nuovo sistema partitico. Allo stesso tempo però è probabile che si pongano all'ordine del giorno anche nuovi problemi derivanti dalle condizioni specifiche (interne o internazionali) del periodo storico durante il quale la democratizzazione prende piede. Un mutato contesto internazionale, le necessità della ricostruzione o della riforma economica, la definizione o attuazione del nuovo sistema istituzionale porteranno sull'agenda politica temi inediti.

Il passaggio a questa seconda fase è spesso accompagnato $\mathrm{da}$ fenomeni come l'apparizione di nuovi partiti o, quantomeno, significative variazioni nel seguito elettorale e mutamenti nell'identità ideologica e programmatica di quelli esistenti, scissioni o fusioni di partiti, nuove formule coalizionali. Si tratta di risposte alla nuova agenda politica e di indicatori di uno spazio politico in trasformazione. Tuttavia non ci dobbiamo aspettare che in tutti i casi la ristrutturazione dello spazio politico sia ugualmente significativa. Là dove l'effetto transizione era stato meno forte dovrebbero essere minori anche le differenze tra le due fasi (tab. 3).

I due problemi principali da considerare a questo punto sono: (a) quali fattori contribuiscono a definire la struttura dello spazio politico e del sistema partitico dopo la fase di transizione; $(b)$ in che misura e con che rapidità la nuova struttura si consoliderà.

Se cominciamo dai casi dell'Europa occidentale possiamo facilmente notare rilevanti differenze sia sotto il profilo del grado di cambiamento che intercorre tra le due fasi sia quanto alla velocità del consolidamento del sistema partitico.

Tra i paesi nei quali la transizione aveva inciso profondamente sull'assetto del sistema partitico, l'Italia mostra assai presto i segni di una profonda ristrutturazione post-transizione del- 
TAB. 3. Mutamenti principali a livello di sistema o di unità partitiche prima o in corrispondenza delle diverse tornate elettorali

\begin{tabular}{|c|c|c|c|}
\hline & \multicolumn{3}{|c|}{ Elezioni } \\
\hline & Seconde & Terze & Quarte \\
\hline Austria & $\begin{array}{l}\text { cambiamento coalizionale } \\
\text { limitato }\end{array}$ & - & - \\
\hline Francia & $\begin{array}{l}\text { importante cambiamento } \\
\text { coalizionale }\end{array}$ & $\begin{array}{l}\text { nuovo partito } \\
\text { di media grandezza }\end{array}$ & $\begin{array}{l}\text { nuovo partito } \\
\text { di media grandezza }\end{array}$ \\
\hline Germania & $\begin{array}{l}\text { assorbimento progressivo } \\
\text { dei partiti minori }\end{array}$ & $\begin{array}{l}\text { assorbimento progressivo } \\
\text { dei partiti minori }\end{array}$ & - \\
\hline Italia & $\begin{array}{l}\text { importante scissione } \\
\text { importante cambiamento } \\
\text { coalizionale }\end{array}$ & - & - \\
\hline Grecia & $\begin{array}{l}\text { declino di un partito di } \\
\text { media grandezza/ascesa di } \\
\text { un partito medio a partito } \\
\text { di prima grandezza }\end{array}$ & alternanza al governo & - \\
\hline Portogallo & $\begin{array}{l}\text { incertezza nelle formule } \\
\text { coalizionali }\end{array}$ & $\begin{array}{l}\text { incertezza nelle } \\
\text { formule coalizionali }\end{array}$ & \\
\hline Spagna & - & $\begin{array}{l}\text { collasso del partito } \\
\text { maggiore/ alternanza al } \\
\text { governo }\end{array}$ & - \\
\hline Bulgaria & $\begin{array}{l}\text { fratture nell'alleanza } \\
\text { partitica/mutamento } \\
\text { coalizionale }\end{array}$ & & \\
\hline Polonia & $\begin{array}{l}\text { disintegrazione del partito } \\
\text { principale/incertezze } \\
\text { coalizionali }\end{array}$ & $\begin{array}{l}\text { risurrezione dei partiti } \\
\text { post-comunisti }\end{array}$ & \\
\hline Rep. Ceca & $\begin{array}{l}\text { scissione del partito maggiore } \\
\text { formazione di un grande partito }\end{array}$ & - & \\
\hline Rep. Slovacca & $\begin{array}{l}\text { collasso del partito maggiore } \\
\text { formazione di un grande partito }\end{array}$ & nuova coalizione & \\
\hline Romania & $\begin{array}{l}\text { scissione nel movimento- } \\
\text { guida della transizione } \\
\text { formazione alleanza dei } \\
\text { partiti di oppposizione }\end{array}$ & & \\
\hline Ungheria & $\begin{array}{l}\text { scissioni nei partiti di } \\
\text { governo/ritorno dei partiti } \\
\text { postcomunisti/ alternanza al governo }\end{array}$ & & \\
\hline
\end{tabular}

lo spazio politico. La prima sfida all'assetto politico centrato sulla coalizione anti-fascista viene dalla apparizione sulla destra di nuovi partiti, come quello dell'Uomo Qualunque e quello 
Monarchico. Questi partiti non sono direttamente legati al passato non democratico, tuttavia definiscono la propria identità in opposizione alla alleanza anti-fascista. L'uscita del Partito Liberale dalla grande coalizione e il suo spostamento a destra è stato un altro sviluppo significativo e connesso. I due fenomeni combinati assieme hanno contribuito a «scoprire» il fianco al principale partito non di sinistra della coalizione anti-fascista, cioè la Democrazia Cristiana. Questa situazione di accresciuta competitività ha fatto sentire i suoi effetti non appena il governo si è trovato a dover fare i conti con i problemi della ricostruzione economica. Su questo quadro, già in movimento rispetto alla originaria formula di grande coalizione di tutti i partiti, si è aggiunta poi la grande frattura internazionale tra Est e Ovest che ha spaccato nel vivo la coalizione anti-fascista. Questo cleavage non ha prodotto soltanto una divisione nella coalizione, ma anche in uno dei partiti più importanti di questa, il Partito Socialista. La collocazione rispetto agli schieramenti internazionali (con tutte le implicazioni politiche, economiche e sociali di questi) è diventata a partire da questo punto un elemento cruciale della identità politica della Democrazia Cristiana e la dominanza di questo tema ha fatto rientrare le sfide che avevano cominciato a provenire dalla destra. In questa fase troviamo dunque tutti gli indicatori di una ristrutturazione dello spazio politico: alta volatilità, nuovi partiti, una importante scissione, la ridefinizione delle identità politiche, cambiamenti negli allineamenti coalizionali. Il risultato sarà una strutturazione molto durevole del sistema partitico secondo il ben noto modello basato su coalizioni di centro obbligate e sulla duplice esclusione dei partiti di sinistra, legati allo schieramento internazionale «sbagliato», e dei partiti di destra, critici delle scelte costituzionali originarie. Gli allineamenti coalizionali e di opposizione rimarranno fondamentalmente invariati per i successivi quaranta anni. Gli effetti di questa strutturazione sono molto marcati anche al livello delle unità partitiche: di particolare rilievo è il consolidamento della Democrazia Cristiana come partito nazionale di prima grandezza (Morlino 1991) e dall'altro lato il decadimento del Partito Socialista a partito di secondo rango e con caratteri di notevole fragilità.

E interessante confrontare il caso italiano con quello francese perché, accanto ad alcune importanti somiglianze, ci sono anche rilevanti differenze. Anche in Francia il cleavage internazionale contribuisce a por fine nel 1947 alla coalizione anti-fa- 
scista. In questo caso però la frattura comunismo/anti-comunismo non è stata in grado di produrre una forte strutturazione del sistema partitico. La sfida gollista sulla destra, centrata sulle scelte costituzionali e in particolare sulla questione dell'assetto del potere esecutivo e nata già prima del manifestarsi del conflitto internazionale, ha impedito il consolidarsi di un forte partito di centro-destra come in Italia. Chiaro segno della più debole strutturazione del sistema partitico sono i successi di partiti-meteora (i Gollisti nel 1951 e i Poujadisti nel 1956) e i livelli della volatilità che restano molto alti (tra i più alti in Europa occidentale) per tutti gli anni cinquanta (cfr. tab. 1). Gli allineamenti coalizionali sono determinati in primo luogo dalla esclusione del Partito Comunista e poi dalle linee tradizionali di divisione della società francese (sinistra/destra socioeconomica, secolare/confessionale). Ma i conflitti istituzionali e poi quelli legati al tema della decolonizzazione aggiungono nuove complicazioni a questo paesaggio politico. La frammentazione del sistema partitico e la necessità, per raggiungere una maggioranza parlamentare, di includere nelle coalizioni partiti divisi dai cleavages tradizionali hanno contribuito significativamente alla instabilità governativa (Williams 1958). Solo dopo il 1958, con la Quinta Repubblica e la soluzione ad un tempo dei conflitti istituzionali e coloniali, si è avuta una seconda strutturazione che ha dato al sistema partitico un assetto più stabile.

Il caso austriaco come abbiamo visto segue invece una strada molto particolare. La grande coalizione dei due partiti maggiori non viene infatti abbandonata dopo la transizione ma resta in piedi diventando una caratteristica durevole del sistema politico. Durerà in effetti fino al 1966 e, dopo un periodo di governi monopartitici e un breve esperimento di piccola coalizione, ritornerà nel 1987 (Gerlich e Müller 1983). La spiegazione per lo straordinario successo della formula coalizionale nasce solo in parte dal formato del sistema partitico nel quale solo raramente uno dei due partiti maggiori è stato in grado di raggiungere da solo la maggioranza assoluta in parlamento. Tanto è vero che altre soluzioni teoricamente possibili - governi di minoranza o piccole coalizioni con il terzo partito - sono state scartate. Le ragioni della scelta devono dunque essere cercate altrove e deve trattarsi di ragioni che non hanno perso la loro presa con la fine del periodo di transizione. Forse si può dire che la necessità originaria di far fronte comune per preservare il nuovo stato democratico dalle pericolose rivalità politiche del 
passato si è progressivamente trasformata, in un paese con una identità nazionale debole come l'Austria, in una sorta di identificazione dei due partiti maggiori con la statualità stessa. L'alleanza tra Cattolici e Socialisti, anche se non strettamente obbligatoria ha finito così per essere percepita come la soluzione «naturale» per affrontare i problemi politici del paese.

Nei due casi della Germania e della Grecia, dove le caratteristiche del sistema partitico erano state assai poco influenzate dalle esigenze specifiche della transizione, come ci si poteva aspettare, non si riscontrano negli anni successivi segni di una rilevante ristrutturazione dello spazio politico. In Germania la competizione tra SPD e CDU/CSU è sin dall'inizio l'elemento caratterizzante del sistema partitico. La completa sconfitta del regime nazista e il forte controllo esercitato dalle potenze occupanti hanno creato una situazione nella quale la necessità di una coalizione di tutte le forze democratiche era assai poco stringente e contemporaneamente $i$ rischi per le forze politiche di procedere autonomamente erano ridotti. Per di più la scelta della piena competizione si adattava meglio alla esigenza, comune ai due partiti maggiori, di contrastare la nascita e il rafforzamento di altri partiti in grado di minacciarli sulle ali (a sinistra per la SPD, a destra per la CDU/CSU). Questo punto era particolarmente rilevante per i Cristiano-Democratici; mentre per la SPD la divisione della Germania causata dalla frattura internazionale e l'avvento di una dittatura comunista a Est ha ridotto rapidamente la importanza di una possibile sfida da sinistra della KPD, per la CDU le minacce erano molto più reali. Bisogna tener presente, infatti, che la CDU nasce come una vera e propria coalizione a cavallo delle vecchie divisioni confessionali tra cattolici e protestanti e anche tra centro e destra, e che nei primi anni del dopoguerra spuntano vari altri partiti che guardano allo stesso elettorato. La scelta di una coalizione proprio con alcuni di questi partiti di centro-destra, invece che con la sinistra, corrisponde chiaramente ad una strategia volta a ridurne il potenziale di competizione. Strategia che avrà pieno successo e porterà all'assorbimento nella CDU o alla sparizione di tutti questi partiti con la sola eccezione della FDP. La competizione destra/sinistra tra CDU/CSU e SPD dominerà quindi sempre più il sistema partitico tedesco.

In Grecia il successo della strategia competitiva di Karamanlis e della risposta di Papandreou è evidenziato dalla sparizione dell'Unione di Centro e dalla debolezza della estrema de- 
stra. Il ritorno al potere di Nuova Democrazia dopo le tre elezioni del 1989/90 indica il notevole potenziale di persistenza dell'assetto originario del sistema partitico. E questo si può in larga misura ricondurre al tradizionale antagonismo tra destra e sinistra che è stato incanalato in un sistema quasi bipartitico, liberato però, per effetto della esperienza passata, dalla minaccia degli attori anti-democratici (i militari e la corona).

In Spagna la fine della transizione è seguita a breve distanza dal crollo della UCD; con questa viene meno il predominio di un partito di centro e si afferma il dualismo tra sinistra e destra incarnate dal PSOE e da AP (poi PP). Il crollo della UCD ha certo delle ragioni interne, ma ancor prima sembra da ricondurre a una ristrutturazione dello spazio politico e in particolare all'affermarsi di una competizione moderata tra destra e sinistra dopo che gli spettri delle contrapposizioni passate erano stati esorcizzati grazie alla consensualità della transizione. Proprio il successo pieno della transizione finisce per togliere spazio ad un conglomerato centrista di partiti quale era la UCD. Una volta che il pericolo di un conflitto radicalizzato tra destra e sinistra viene meno, si indeboliscono anche $i$ fattori che avevano fatto convergere e tenuto insieme nella UCD i diversi gruppi. Il crollo di questo partito viene quindi a coincidere (e si potrebbe dire che in parte ne è causato e in parte ne è la causa) con il grande successo elettorale della sinistra moderata (PSOE) e della destra (AP). Per quest'ultimo partito il successo elettorale sarà però accompagnato dalla persistenza di alcuni elementi di debolezza che gli impediranno a lungo di raggiungere da solo la maggioranza o di stringere alleanze con altri partiti. In questo senso ha giocato forse un certo retaggio del passato regime che è rimasto attaccato ad AP. Ma probabilmente ancor più rilevanti sono stati gli effetti del tradizionale cleavage centro/periferia che rendono difficile alla destra (centralistica) accreditarsi nelle regioni a più alto tasso autonomista e allearsi con i partiti regionalisti. Questa asimmetria ha consentito al Partito Socialista di dominare per più di un decennio, con una propria maggioranza o, negli ultimi tempi, grazie ad accordi coalizionali. In conclusione la strutturazione dello spazio politico che emerge in Spagna può essere considerata il risultato, da un lato, dei successi raggiunti nel fare $\mathrm{i}$ conti con le eredità del passato e nel risolvere i problemi della transizione e, dall'altro, dall'affermarsi di un «normale» cleavage destra/sinistra ormai liberato dalle antiche radicalizzazioni e affiancato dal tradizionale cleavage centro/periferia. 
Se passiamo ai paesi dell'Europa centro-orientale ci troviamo di fronte a situazioni caratterizzate ancora da notevole incertezza; dobbiamo limitarci quindi ad esaminare i primi passi del periodo post-transizione.

In Polonia i dati caratterizzanti sono l'«esplosione» di Solidarnosc, la iperframmentazione del sistema partitico e poi il grande ritorno dei partiti post-comunisti alle elezioni del 1993. Nel periodo tra le elezioni del 1991 e del 1993 i processi coalizionali sono particolarmente difficili per una combinazione di fattori. $\mathrm{Da}$ un lato vale ancora come eredità della transizione la esclusione dei partiti post-comunisti (la Socialdemocrazia, principale erede del POUP, e il Partito Contadino) dalle coalizioni di governo (anche se non da maggioranze parlamentari transitorie). D'altro canto la comune derivazione da Solidarnosc degli altri partiti ha perso ormai molta della sua forza aggregante e non può più costituire la base per coalizioni stabili. L'opposizione al vecchio regime è sempre meno rilevante per la definizione dello spazio politico rispetto al duro conflitto istituzionale accesosi tra buona parte dei partiti e il presidente, e ad altre linee di conflitto come quella economica (riforma contro intervento statale) o quelle culturali (confessionalismo vs. secolarizzazione o cosmopolitismo vs. nazionalismo) (Gebethner 1993). Le coalizioni formatesi dopo il 1991 si sono basate prevalentemente su questi elementi. Dopo il governo Olszewski, fondato su una coalizione orientata verso il polo confessionale, nazionalista e (in parte) anti-presidenziale, la coalizione successiva del governo Suchocka ha tentato un compromesso a cavallo delle linee di conflitto culturali combinato con una linea più accomodante con il presidente della repubblica e con il sostegno alla riforma economica. Il fatto che quest'ultima coalizione abbia riunito gran parte dei partiti originati da Solidarnosc non ha significato tuttavia un ritorno alla politica del periodo di transizione. È stato presto chiaro che questi legami non erano sufficienti a tener insieme la coalizione. In meno di un anno i conflitti sui problemi economici (salari del settore pubblico, privatizzazioni, sussidi all'agricoltura) hanno fatto emergere la fragilità della coalizione. L'indebolimento e la crisi del governo Suchocka hanno anche fatto riemergere la tensione tra orientamenti presidenzialisti e parlamentaristi. La decisione di Walesa di creare un proprio movimento per le elezioni del $1993^{11}$ indi-

${ }^{11} \grave{E}$ interessante notare che la sigla di questo partito è la stessa del movimento 
ca che il conflitto istituzionale, un po' come nella Quarta Repubblica francese e in Portogallo fino agli anni ottanta, rimane un elemento non risolto che interferisce con gli allineamenti politici rendendo problematica la stabilizzazione del sistema partitico (e tenendo aperto il dibattito sulla legittimità delle istituzioni democratiche) ${ }^{12}$. Il grande successo elettorale dei partiti postcomunisti (SLD e PSL) e la rotta della maggior parte dei partiti post-Solidarnosc nelle elezioni del 1993 (amplificate entrambe dal nuovo sistema elettorale) hanno trasformato ancora e semplificato il paesaggio politico. Il messaggio più chiaro che ne emerge è che la delegittimazione politica dei regimi comunisti non si riflette più sui partiti-successori che al contrario possono trar vantaggio dallo scontento generato dalla transizione economica. Paradossalmente è proprio perché la transizione politica è finita che la transizione economica con tutti i suoi problemi può diventare il terreno di competizione principale tra $\mathrm{i}$ partiti. $\mathrm{Nel}$ momento in cui di fronte al peso delle preoccupazioni economiche le altre linee di conflitto perdono di rilevanza, i partiti post-comunisti hanno potuto presentarsi come i rappresentanti di interessi molto diffusi che si sentivano minacciati dalla rapidità e profondità della riforma economica. D'altro canto le forze favorevoli alla riforma economica rimangono divise al loro interno da conflitti culturali e istituzionali e non sono in grado di costituire un fronte compatto.

A paragone della Polonia il caso ceco sembra aver seguito una linea di sviluppo più semplice. Il passaggio al dopo-transizione è stato ancora più netto; la rapida crisi del fronte anti-comunista ha lasciato posto in questa metà della vecchia Cecoslovacchia ad una organizzazione dello spazio politico molto più semplice che nella metà slovacca. L'assenza o comunque la limitata rilevanza del problema istituzionale della presidenza, dovuta sia al meno forte ruolo personale di Havel rispetto a quello di Walesa sia alla maggiore chiarezza delle scelte costituzionali $^{13}$, e la debolezza dei conflitti culturali hanno consentito un

creato da Pilsudski (il Blocco apartitico per la cooperazione con il governo) nel 1927 come strumento di legittimazione elettorale della sua leadership autoritaria (Rothschild 1990, 59).

${ }_{12}$ Per una attenta discussione dei conflitti potenziali tra soluzioni presidenziali e consolidamento democratico si vedano le analisi di Linz (1994).

${ }_{13}$ Non è un caso che il processo di elaborazione costituzionale raggiunga la sua conclusione più rapidamente e con maggiore facilità in Cecoslovacchia che in Polonia. Le questioni costituzionali escono nel primo paese rapidamente dalla scena. 
grado minore di frammentazione dei partiti derivati dalla dissoluzione del Foro Civico ${ }^{14}$. L'asse economico destra/sinistra è emerso come quello dominante e il Partito Democratico Civico di Klaus, che si è identificato chiaramente con il polo orientato alla riforma di mercato dell'economia, ha potuto ereditare buona parte del seguito del Foro Civico. Il governo Klaus trova il suo sostegno in una coalizione di centro-destra che include anche due piccoli partiti democristiani e si trova di fronte una sinistra divisa in due campi - da un lato il Partito Comunista, dall'altro il «Blocco realistico» (una alleanza nata da poco tra Socialdemocratici, Unione Liberale Sociale e altri gruppi minori). La situazione è dunque praticamente l'opposto di quella polacca: nella repubblica ceca il fronte che propugna la riforma economica è forte e unito, in quella polacca è invece diviso; viceversa il fronte dell'interventismo statale è debole a Praga e forte a Varsavia. Il tema è ugualmente importante per definire gli allineamenti politici ma opposta è la parte vincente. La differenza tra i due paesi sembra da ricondursi al fatto che nella Repubblica Ceca le altre linee di conflitto hanno avuto un'incidenza minore sulle forze orientate alla riforma economica. Rimane incerto ad oggi se la alleanza dei partiti della sinistra non comunista, da un lato, e quella dei partiti al governo, dall'altro, siano destinate a conservare il carattere di alleanze di partiti con una identità distinta o invece possano condurre ad una fusione o assorbimento dei singoli partiti in unità più ampie.

Nella Slovacchia il processo di definizione dello spazio politico sembra invece assai meno avanzato. Dopo la transizione dal comunismo il paese ha affrontato anche una transizione «dalla Cecoslovacchia» che ha fortemente contribuito ad una nuova strutturazione del sistema partitico. L'improvviso spostamento dell'attenzione politica dal problema dell'uscita dal comunismo a quello nazionale ha rapidamente messo fuori gioco il movimento anticomunista (Pubblico contro la Violenza), seriamente danneggiato il Partito Cristiano Democratico e favorito la crescita spettacolare del nuovo movimento nazionalista (lo HZDS di Meciar). Gli altri partiti, come il post-comunista Partito della Sinistra Democratica e, abbastanza comprensibilmen-

\footnotetext{
${ }^{14}$ Non si ha nulla di paragonabile al conflitto che si delinea in Polonia tra orientamenti nazionalisti e «cosmopoliti»; inoltre la maggiore debolezza della Chiesa cattolica rende il cleavage clericale/anticlericale meno rilevante.
} 
te, il Partito Nazionale Slovacco e la coalizione della minoranza ungherese, sono stati invece molto meno colpiti dal mutamento dell'agenda politica. Gli allineamenti coalizionali sono stati determinati essenzialmente dalla issue nazionale. I Cristiano Democratici e il partito della minoranza ungherese, che erano più tiepidi o addirittura si opponevano alla secessione da Praga, hanno fatto fronte comune; e anche il Partito della Sinistra Democratica dopo un iniziale sostegno a Meciar è passato alla opposizione. Il Partito Nazionale Slovacco ha seguito invece una linea di condotta più incerta; dopo aver sostenuto il governo Meciar si è alleato con l'opposizione per poi tornare dopo poco a fianco del governo. $\mathrm{Ma}$, una volta raggiunto l'obiettivo principale del movimento nazionalista, i dissensi sono presto emersi nel partito di Meciar provocandovi delle scissioni. L'unione di tutte le opposizioni dalla sinistra ai Cristiano Democratici agli scissionisti del movimento nazionalista ha alla fine determinato la caduta del governo Meciar. Questa eterogenea coalizione indica che la linea di frattura tra nazionalisti e anti-nazionalisti rimane quella caratterizzante per il sistema politico. È cambiata soltanto la parte vincitrice. D'altra parte, i problemi di minoranze e di confini con la Ungheria e ora anche con la Repubblica Ceca tengono in vita questa tematica e nel giro di pochi mesi (nell'autunno 1994) il fronte nazionalista riprenderà la guida del paese.

L'Ungheria sembrava essere entrata molto più precocemente degli altri paesi dell'area nella fase politica post-transizione. Come si è visto la prima coalizione di governo era stata determinata assai più dalle linee di conflitto che dividevano tra loro $\mathrm{i}$ partiti anti-comunisti che dall'opposizione al vecchio regime. In buona misura si è trattato di un ritorno dei cleavages tradizionali dell'Ungheria: i valori cristiani e nazionali contro le posizioni liberali e secolaristiche. Se si aggiunge l'antagonismo centro/periferia tra Budapest e la provincia, abbiamo le basi per la divisione tra la coalizione di governo del Foro Democratico in unione con il Partito dei Piccoli Proprietari e la Democrazia Cristiana e l'opposizione dei partiti liberali (Korosenyi 1993). Tuttavia gli ultimi mesi dei governi Antall e Boross e poi le elezioni del 1994 mostrano che anche qui il quadro politico non è ancora stabilizzato. La coalizione di governo e in specie il suo partito guida hanno dovuto far fronte a forti tensioni interne. Le discussioni sulla restituzione delle terre hanno indotto la leadership dei piccoli proprietari ad abbandonare la coalizione 
(febbraio 1992) e ne è derivata anche una scissione nel partito (su quarantacinque deputati trentacinque sono però rimasti fedeli al governo). Nel Foro Democratico l'ala nazionalista è diventata estremamente sospettosa di ogni mossa del partito verso il centro e di una possibile collaborazione con uno dei due partiti liberali (cioè l'Alleanza dei Giovani Democratici). Alla fine il leader di questa ala, Czurka, ha abbandonato il partito creandone uno nuovo - il Partito della Giustizia. Per un po' è parso che l'esito più probabile sarebbe stato quello di un sistema partitico tripolare con al centro i partiti liberali e i resti del Foro Democratico, a sinistra il partito post-comunista e a destra quello nazionalista. Ma le elezioni del 1994 hanno prodotto una situazione abbastanza simile a quella polacca; la divisione dell'area non comunista e i costi della transizione economica hanno favorito il successo del partito post-comunista. Questo, che è diventato (anche grazie al sistema elettorale) di gran lunga il partito maggiore, può contare sulla collaborazione del più grande dei partiti liberali, mentre il centro-destra resta diviso in quattro partiti più piccoli (Giovani Democratici, Foro, Cristiano Democratici e Piccoli Proprietari).

I casi bulgaro e romeno restano infine ancora difficili da decifrare. La sopravvivenza senza vere rotture di continuità di forze politiche di prima grandezza legate al vecchio regime solleva problemi interessanti. $\mathrm{Ci}$ si potrebbe addirittura chiedere se la transizione alla democrazia sia veramente compiuta. In questo caso dovremmo attenderci nel prossimo futuro un profondo riassetto delle forze politiche. Oppure dobbiamo ritenere che il Partito Socialista Bulgaro e il Fronte Democratico di Salvezza Nazionale siano riusciti a ridefinire la loro identità politica in senso democratico, trasformandosi in partiti «normali» che rappresentano interessi comuni, e abbiano cessato di essere strumenti per cercare di preservare il passato? Se questo è vero ci si deve chiedere che cosa accadrà alla identità politica di quei conglomerati di partiti che erano tenuti uniti dalla contrapposizione al vecchio regime. In effetti ad uno sguardo più attento si scorgono dietro la apparente continuità della linea di conflitto pro/contro il vecchio regime segni di un nuovo spazio politico in via di definizione.

In Romania il Fronte di Salvezza Nazionale si è diviso nel corso del 1992. Dopo non molto tempo le sue due ali erano già in cerca di alleanze. Il Fronte Democratico di Iliescu ha stabilito un'intesa sempre più salda con i partiti della estrema destra 
nazionalista (il Partito della Grande Romania e il Partito dell'Unità Nazionale Romena) che dal novembre 1992 hanno dato il loro sostegno al governo Vacaroiu. E nel luglio 1993 ha cambiato il nome in quello di Partito della Socialdemocrazia di Romania. Dall'altro lato, il Fronte dell'ex primo ministro Petre Roman si è prima fuso con il piccolo Partito Democratico, assumendo la denominazione di Partito Democratico-Fronte di Salvezza Nazionale, e poi si è avvicinato alla Convenzione Democratica Romena. Il panorama politico, per quanto ancora ampiamente condizionato dai forti elementi di continuità con il passato, sta dunque prendendo forma incorporando aspetti nuovi. Allineamenti e coalizioni rispondono in parte a problemi connessi all'eredità del vecchio regime (processi di decomunistizzazione, privatizzazioni, ecc.), ma anche a cleavages indipendenti dalla transizione come quello etnico e nazionale.

Anche in Bulgaria stanno emergendo elementi di una politica di post-transizione. A differenza che in Romania il fronte di opposizione (l'Unione delle Forze Democratiche) ha ottenuto più presto l'accesso al potere di governo (oltre ad aver piazzato uno dei suoi uomini alla guida dello Stato). Si potrebbe forse dire che il governo Dimitrov, formato da uomini dell'Unione e sostenuto anche dal partito della minoranza turca, ha ricalcato in buona misura il modello della grande coalizione di opposizione al vecchio regime. L'unità di questa grande coalizione ha cominciato però presto a mostrare le crepe. Alcuni partiti come il Partito Agrario Petkov e il Partito Socialdemocratico avevano lasciato già dal 1991 l'Unione. Ma i conflitti sono diventati ancora più seri durante il governo Dimitrov; i contrasti tra il presidente Zhelev e la leadership dell'Unione e le ulteriori scissioni hanno finito per far cadere il primo governo delle opposizioni. Dal dicembre 1992 è seguito un governo tecnocratico guidato da Berov che ha potuto godere del sostegno di una coalizione inaspettata composta dal Partito Socialista Bulgaro, dal Partito della minoranza turca e dalla Nuova Unione per la Democrazia, ala scissionista della Unione delle Forze Democratiche. Un insieme di rivalità personali e di divergenze più sostanziali concernenti le politiche economiche, fiscali e religiose sembra essere stato alla base di questa ristrutturazione del sistema partitico. Stupisce in particolare la collaborazione tra il Partito Socialista e il Partito turco, data la posizione fortemente nazionalista e di opposizione all'autonomia per le minoranze etniche tenuta dal primo durante la campagna elettorale. La posizione del Partito 
della minoranza turca può essere probabilmente interpretata come il tentativo di acquisire una posizione pivotale tra le due forze politiche principali al fine di far valere meglio le rivendicazioni del proprio elettorato (Karasimeonov 1993). Anche qui, dunque, stanno emergendo problematiche nuove.

\section{Conclusioni}

L'esame dei casi, tanto dell'Europa occidentale che di quella centro-orientale, ha confermato con sufficiente chiarezza quanto ci si aspettava, cioè che nei primi anni dopo il ritorno alla democrazia i mutamenti tanto a livello di unità partitiche che di sistema dei partiti sono in genere assai rilevanti (tab. 3). La nascita di nuovi partiti, le scissioni e fusioni di partiti già esistenti, la ridefinizione delle identità originarie, i mutamenti negli schemi coalizionali sono fenomeni all'ordine del giorno. La frequenza di questi eventi mostra piuttosto chiaramente che con il passaggio dalla transizione al dopo-transizione la organizzazione dello spazio politico attraversa un processo di trasformazione.

I risultati dell'analisi dei casi possono essere sintetizzati nei seguenti punti:

1. La prima strutturazione del sistema partitico nelle nuove democrazie è in genere profondamente influenzata dai problemi speciali che devono essere affrontati durante la transizione. La necessità di unire le forze per rimuovere il regime non democratico, creare una base di sostegno sufficiente per il nuovo regime ed esorcizzare gli spettri dei passati fallimenti della democrazia tende a favorire la nascita di formazioni politiche ad hoc e/o di relazioni speciali tra partiti. Fronti di opposizione o movimenti nei quali convergono orientamenti ideologici anche assai diversi e grandi coalizioni sono la risposta tipica a queste situazioni.

2. Ci sono però alcune importanti eccezioni a questa «legge». Quando l'impatto specifico del periodo di transizione è meno pronunciato, la strutturazione del sistema partitico sarà fin dall'inizio determinata da altri fattori. La spiegazione di queste eccezioni deriva dal fatto che in questi casi la transizione alla democrazia non costituisce un problema speciale per i partiti, o perché fortemente controllata dall'esterno o perché realizzatasi con più rapidità e semplicità. I casi più rilevanti sono 
quelli della Germania, della Grecia e almeno in parte dell'Ungheria.

3. La prima strutturazione del sistema partitico favorita dalle esigenze della transizione è per sua natura temporanea. La soluzione dei problemi specifici di questa fase fa sì che quegli aspetti del sistema partitico (formule coalizionali e/o formazioni politiche) che si erano sviluppati per darvi risposta perdono sempre più la loro forza e più facilmente vengono sottoposti a critica. Lo spazio politico si amplia e acquista dimensioni nuove. Si manifestano livelli piuttosto alti di volatilità e la ricerca di nuovi allineamenti diventa una preoccupazione centrale dei leader politici. Questo è il momento propizio per la nascita di nuovi partiti, per il manifestarsi di fratture all'interno dei vecchi e per l'esplodere di crisi nelle coalizioni.

4. La nuova strutturazione del sistema partitico che si definisce dopo la fase di transizione riflette una combinazione tra effetti del passato (le eredità delle precedenti esperienze democratiche, ma anche del regime non democratico e i cleavages socio-politici di lunga durata) e quelli delle nuove issues e dei conflitti che emergono sulla scena al momento della ridemocratizzazione.

5. Somiglianze interessanti possono essere individuate tra $i$ casi riferibili alle tre ondate di democratizzazioni che abbiamo esaminato. Tuttavia i paesi dell'Europa centro-orientale presentano alcuni caratteri peculiari. La debolezza dei vecchi partiti democratici rende sin dall'inizio più difficile la strutturazione del sistema partitico. I colossali problemi della transizione economica e sociale dall'economia di stato all'economia di mercato, che non si esauriscono con la transizione politica, facilitano un fenomeno che non ha riscontri (paragonabili) nei casi occidentali: la ripresa dei partiti legati al regime non democratico.

6. Nei casi per i quali possiamo disporre di un periodo di osservazione abbastanza lungo, cioè per i paesi della prima ondata e, seppur in misura minore, per quelli della seconda possiamo osservare che la strutturazione del sistema partitico, dopo aver metabolizzato il periodo di transizione, è stata piuttosto rapida e con effetti di lungo termine. Dove ciò non è accaduto come nella Francia della Quarta Repubblica - la instabilità è derivata in buona misura dai dissensi originari circa la legittimazione delle istituzioni democratiche. L'esistenza di problemi simili in uno dei paesi dell'Europa centro-orientale che abbiamo discusso (come la Polonia) e in altri che qui non sono stati 
affrontati (ad es. la Russia) indica che anche su questo punto si possono trovare parallelismi significativi tra le due parti dell'Europa.

\section{Riferimenti bibliografici}

Agh, A. (1992), The Parliamentary Way to Democracy: the Case of Hungary, Budapest Papers on Democratic Transition n. 2, Budapest, Budapest University of Economics.

Bartolini, S. e P. Mair (1990), Identity, Competition and Electoral Availability, Cambridge, Cambridge University Press.

Bluhm, W.T. (1973), Building an Austrian Nation. The Political Integration of a Western Nation, New Haven, Yale University Press.

Budge, I. e H. Keman (1990), Parties and Democracy. Coalition Formation and Government Functioning in Twenty States, Oxford, Oxford University Press.

Burton, M., R. Gunther e J. Higley (1992), Introduction: Elite Transformations and Democratic Regimes, in J. Higley e R. Gunther (a cura di), Elites and Democratic Consolidation in Latin America and Southern Europe, Cambridge, Cambridge University Press.

Cotta, M. (1993), New Party Systems after the Dictatorship: Dimensions of Analysis. The East European Cases in a Comparative Perspective. Working Paper n. 9, Dipartimento di Scienze Storiche, Giuridiche, Politiche e Sociali, Università di Siena.

- (1994), Building Party Systems after the Dictatorship. The East European Cases in a Comparative Perspective, in G. Pridham e T. Vanhanen (a cura di), Democratization in Eastern Europe, London, Routledge.

Diamandouros, N. (1986), Regime Change and the Prospects for Democracy in Greece: 1974-1983, in G. O' Donnell, Ph. C. Schmitter e L. Whitehead (a cura di), Transitions from Authoritarian Rule. Southern Europe, Baltimore, The Johns Hopkins University Press.

Downs, A. (1957), An Economic Theory of Democracy, New York, Harper \& Row.

Engelmann, F.C. (1966), Austria: Pooling the Opposition, in R. Dahl (a cura di), Political Oppositions in Western Democracies, New Haven, Yale University Press.

Gebethner, S. (1993), Political Parties in Poland after the Semi-Free Elections of 1989, mimeo.

Gerlich, P. e W.C. Müller (a cura di) (1983), Zwischen Koalition und Konkurrenz. Oesterreichs Parteien seit 1945, Wien, Braumueller.

Grilli di Cortona, P. (1989), Le crisi politiche nei regimi comunisti, Milano, Franco Angeli.

- (1994), Nuovi conflitti e nuovi sistemi partitici all'Est. Verso un 
modello esplicativo, in «Rivista Italiana di Scienza Politica», XXIV, pp. 203-236.

Gunther, R. (1992), Spain: the Very Model of the Modern Elite Settlement, in J. Higley e R. Gunther (a cura di), Elites and Democratic Consolidation in Latin America and Southern Europe, Cambridge, Cambridge University Press.

Gunther, R., G. Sani e G. Shabad (1988), Spain After Franco. The Making of a Competitive Party System, Berkeley, University of California Press.

Huneeus, C. (1985), La Unión de Centro Democrático y la transición a la democracia en España, Madrid, Centro de Investigaciones Sociológicas.

Karasimeonov, G. (1993), Sea Changes in the Bulgarian Party System, in «The Journal of Communist Studies», IX.

Kitschelt, H., (1992), The Formation of Party Systems in East Central Europe, in «Politics and Society», XX.

Korosenyi, A. (1993), Stable or fragile Democracy? - Party System in Hungary, in «Government and Opposition», XXVIII, pp. 87-104.

Laver, M. e N. Shofield (1990), Multiparty Government, Oxford, Oxford University Press.

Linz, J.J. (1994), Presidential or Parliamentary Democracy: does it Make a Difference?, in J. Linz e A. Valenzuela (a cura di), The Failure of Presidential Democracy. Comparative Perspectives, Baltimore, The Johns Hopkins University Press.

Mackie, T. e R. Rose (1991), The International Almanach of Electoral History, London, Macmillan.

Morlino, L. (1986), Consolidamento democratico: definizione e modelli, in «Rivista Italiana di Scienza Politica», XVI, pp. 197-238.

Morlino, L. (a cura di) (1991), Costruire la democrazia, Bologna, Il Mulino.

Morlino, L. (1995), Parties and Democratic Consolidation in Southern Europe, in R. Gunther, N. Diamandouros e H. J. Puhle (a cura di), The Politics of Democratic Consolidation in Southern Europe, Baltimore, The Johns Hopkins University Press.

Pasquino, G. (1986), The Demise of the First Fascist Regime and Italy's Transition to Democracy: 1943-1948, in G. O' Donnell, Ph. C. Schmitter e L. Whitehead (a cura di), Transitions from Authoritarian Rule. Southern Europe, Baltimore, The Johns Hopkins University Press.

Pridham, G. (1977), Christian Democracy in Western Germany. The CDU/CSU in Government and Opposition, London, Croom Helm.

Radio Free Europe/Radio Liberty, Research Reports, anni 1990-94.

Rothschild, J. (1990), East Central Europe between the Two World Wars, Seattle, University of Washington Press.

Ruhl, H.G. (a cura di) (1982), Neubeginn und Restauration, Muenchen, DTV. 
Sartori, G. (1976), Parties and Party Systems, Cambridge, Cambridge University Press.

Smith, G. (1979), Democracy in Western Germany. Parties and Politics in the Federal Republic, London, Heinemann.

Szajkowski B. (a cura di) (1991), New Political Parties of Eastern Europe and the Soviet Union, Harlow, Longman.

Wandruska, A. (1954), Öesterreichs politische Struktur. Die Entwiklung der Parteien und politische Bewegungen, in H. Benedikt (a cura di), Geschichte der Republik Öesterreichs, Wien.

Williams, Ph. (1958), Politics in Post-war France. Parties and the Constitution in the Fourth Republic, London, Longmans. 